\section{Enhancing the selectivity of kinase inhibitors in oncology: a chemical biology perspective}

\author{
"...detailed bioinformatics analyses of the kinome has helped \\ medicinal chemists recognize subtle differences and rare events \\ among kinase active sites that can be targeted to increase and direct \\ the selectivity of a promiscuous type-I scaffold."
}

\begin{abstract}
First draft submitted: 18 December 2015; Accepted for publication: 22 December 2015; Published online: 22 February 2016
\end{abstract}

Keywords: chemical probe $\bullet$ drug discovery $\bullet$ kinase inhibition $\bullet$ oncology $\bullet$ target selectivity

\begin{abstract}
Aberrant kinase activity has been implicated in numerous diseases, particularly throughout oncology. This has led to an intense effort to develop effective kinase inhibitors that has resulted in a growing number of US FDA-approved drugs [1]. While these efforts have in part revolutionized cancer therapy, a large degree of kinase active site conservation throughout the kinome (over 500 kinases) has rendered the majority of small-molecule kinase inhibitors quite promiscuous [2], leading to off-target kinase inhibition and associated side effects. Kinase inhibitors are often used as tools for target validation and to study the underpinnings of a cellular process or disease. These fundamental studies are often hindered by a lack of kinase inhibitor selectivity that complicates experimental design and analysis [3].
\end{abstract}

There are several classes of kinase inhibitors that target varying conformations or allosteric sites of a kinase. The majority of kinase inhibitors that have been developed bind the kinase active site in a manner similar to ATP, and are known as type-I kinase inhibitors. Many common type-I core scaffolds are based on heterocycles that 'mimic' adenine and form highly analogous interactions with the kinase active site, leading to a significant degree of kinase promiscuity. For this commentary, we will focus on 'chemical biological' strategies toward increasing the selectivity of type-I kinase inhibitors to give new chemical probes. We refer to an excellent review [4] that was recently published for a more general overview on efforts toward more selective kinase inhibitors, many of which are a result of extensive medicinal chemistry and well-executed structural biology.

There has been a great deal of recent progress toward increasing the selectivity of type-I kinase inhibitors. This has been in part driven by the development of new technologies that allow for the rapid evaluation of kinase inhibitor selectivity [5]. In addition, detailed bioinformatics analyses [6] of the kinome has helped medicinal chemists recognize subtle differences and rare events among kinase active sites that can be targeted to increase and direct the selectivity of a promiscuous type-I scaffold. Chemical strategies to exploit these differences have been referred to as 'selectivity filters' by Shokat [7].

The first widely recognized selectivity filter was the gatekeeper residue [8], a single position within the ATP binding site that can either allow access (when the residue is small) or block access (when the residue is bulky) to a hydrophobic pocket in the back of the kinase active site. Medicinal chemists have designed heterocyclic scaffolds that possess a bulky substituent oriented toward the gatekeeper residue, resulting in a steric clash with large gatekeeper residues that prevents binding, while maintaining activity toward kinases with small gatekeeper residues. As an estimated $20 \%$ of kinases have a small gatekeeper, this is an effective strategy to begin to increase the kinase selectivity of a molecule. However, more structural optimization
Sean Maddox

Department of Chemistry \& Biochemistry, San Diego State University, 5500 Campanile Drive, San Diego, CA 92182-1030, USA

David Hecht

School of Mathematics, Science \& Engineering, Southwestern College, 900 Otay Lakes Road, Chula Vista, CA 91910, USA

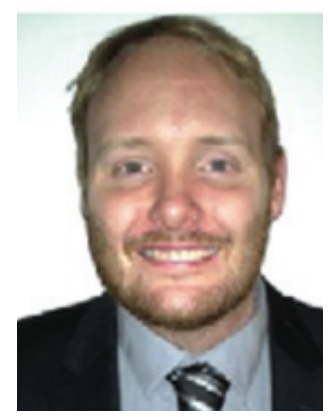

Jeffrey L Gustafson Author for correspondence: Department of Chemistry \& Biochemistry, San Diego State University, 5500 Campanile Drive, San Diego, CA 92182-1030, USA jgustafson@mail.sdsu.edu 
is typically needed to obtain the degree of selectivity needed for chemical probes and pharmaceutical leads.

Another strategy that has benefited from the bioinformatic analysis of the kinase active site is the covalent targeting of a nonconserved cysteine [7,9,10]. This involves the design of inhibitors that possess a weak electrophile (most commonly an acrylamide or an $\alpha$-haloketone) orientated toward a nonconserved cysteine such that the cysteine's sulfur can form a covalent interaction with the inhibitor, drastically increasing the potency and selectivity toward kinases with a cysteine at that position. As nonconserved cysteines at a particular position in the kinase active site are quite rare, covalent targeting has led to exceptionally selective kinase inhibitors, such as recently FDA-approved drugs ibrutinib (mantle cell lymphoma) [11] and osimertinib (EGFR T790M positive non-small-cell lung cancer) [12]. While undeniably successful, this approach relies on the rare event of an accessible nonconserved cysteine in the target's active site, rendering the majority of kinases not amenable. Finally, the reliance of these inhibitors on a covalent interaction with a nonconserved cysteine renders these inhibitors susceptible to drug resistance via mutation of the cysteine to another residue, an event that has already been observed in patients on ibrutinib [13].

\section{"In essence we were able to decouple the various activities of a promiscuous kinase inhibitor to specific atropisomer conformations."}

Another promising strategy to increase type-I inhibitor selectivity is to tether it to a second moiety (small molecule or peptide) that targets another site of the kinase to give bivalent inhibitors [14]. Soellner [15] has recently applied this approach to a promiscuous pyrrazolopyrimidine based inhibitor, linking it to a peptide sequence from an Src substrate, to yield the most selective Src inhibitors reported to date. This strategy holds great promise as a modular approach toward highly selective chemical probes to study the roles of a kinase in cell-signaling processes, however any clinical development will likely suffer from the typical drawbacks of peptide based inhibitors. To begin to overcome these limitations Soellner has recently developed an enzyme template based approach toward the discovery of small molecule bivalent kinase inhibitors [16]. This fragmentbased approach utilizes a thiol containing type-I inhibitor that can react with acrylamide fragments that bind outside the ATP pocket, and has yielded several new small molecule bivalent inhibitors with improved selectivities compared with the original 'parent' scaffold.

We have recently disclosed a new strategy toward increasing the selectivity of kinase inhibitors trough the exploitation of atropisomeric conformation [17]. Atropisomerism is a form of chirality that arises from hindered rotation around a bond in which the rotational conformations are nonsuperimposable mirror images or enantiomers. Atropisomers can exist as stable isolable enantiomers or as rapidly interconverting racemic mixtures depending on the degree of hindrance to rotation about this bond. This dynamic nature coupled with the fact that atropisomers can possess markedly different biological activities has led some in medicinal chemistry to refer to atropisomerism as a 'lurking menace in drug discovery' [18]. Despite this, rapidly interconverting atropisomerism is ubiquitous throughout drug discovery, in part due to the prevalence of chemistries (i.e., Suzuki couplings and amide formations) that can lead to atropisomeric axes [19]. While these compounds are typically treated as achiral molecules, they actually interact with their target in single atropisomer form, with the other atropisomer contributing very little to the relevant activities, and in many cases leading to off-target activities.

This led us to postulate that atropisomerically stable and pure analogs of interconverting atropisomeric biologically active small molecules would have increased selectivity through preclusion of the nonrelevant atropisomer and associated off-target activities. We decided to initially test this hypothesis in the context of kinase inhibitors as selectivity is still an issue and atropisomerism is ubiquitous throughout many common kinase-inhibiting scaffolds. We began these studies on the pyrrolopyrimidine (PPY) scaffold, a common and promiscuous class of kinase inhibitors. The PPY atropisomeric axis arises from an aryl substituent that is designed to target the gatekeeper residue, and thus is selective for the roughly $20 \%$ of kinases with small gatekeeper residues. Additionally, as there were crystal structures in the literature of PPYs bound to kinases in different atropisomeric conformations, PPY's represented an intriguing arena to test whether controlling the atropisomeric conformation would lead to further gains in selectivity.

We were able to quickly access atropisomerically rigidified PPY analogs using our recently developed Lewis base catalyzed halogenation [20] to incorporate substitution adjacent to the interconverting axis. We then separated each atropisomer via HPLC on a chiral stationary phase and assigned each atropisomers stereochemical conformation through small molecule X-ray crystallography and cyclic dichroism. We subjected each atropisomer, as well as a rapidly interconverting 'parent compound' to a panel of tyrosine kinases known to be inhibited by PPYs. While the atropisomeric analogs were less potent than the parent compound, presumably due to steric and electronic issues 
brought about by the incorporated halogen substitution, the atropisomeric analogs displayed increased target selectivity across the panel. Most excitingly, each atropisomer inhibited different kinases, for example, the $(R)$-atropisomer inhibited Ret kinase but not Src or Abl, whereas the $(S)$-atropisomer inhibited Src and $\mathrm{Abl}$, but not Ret, and the interconverting parent molecule inhibited all three. In essence we were able to decouple the various activities of a promiscuous kinase inhibitor to specific atropisomer conformations.

While exciting preliminary results, these analogs need further structural optimization to possess the combination of selectivity and potency needed to be useful chemical probes or pharmaceutical lead compounds, and we currently feel that this approach will be most successful as part of a larger medicinal chemical campaign in combination with other strategies for improving selectivity. We are currently testing this strategy by optimizing the $(R)$-atropisomer for selectivity and potency toward Ret, a validated oncogene in several thyroid cancers including papillary thyroid cancer for which no selective inhibitor exists in the literature. Finally, as atropisomerism is common throughout drug discovery, the control of atropisomeric conformation should be applicable to a wide range of promiscuous scaffolds toward diverse targets beyond kinase inhibitors.

Kinase inhibition is firmly entrenched as a key strategy toward the targeted treatment of many different cancers. A general lack of inhibitor selectivity among kinases is one of the major issues facing oncology drug

\section{References}

Papers of special note have been highlighted as:

- of interest; $\bullet \bullet$ of considerable interest

1 Zhang J, Yang PL, Gray NS. Targeting cancer with small molecule kinase inhibitors. Nat. Rev. Cancer 9(1), 28-39 (2009).

2 Davis MI, Hunt JP, Herrgard S et al. Comprehensive analysis of kinase inhibitor selectivity. Nat. Biotechnol. 29(11), 1046-1051 (2011).

3 Bain J, Plater L, Elliott $\mathrm{M}$ et al. The selectivity of protein kinase inhibitors: a further update. Biochem. J. 408, 297-315 (2007).

4 Müller S, Chaikuad A, Gray NS, Knapp S. The ins and outs of selective kinase inhibitor development. Nat. Chem. Biol. 11(11), 818-821 (2015).

-• Discusses several recent examples of increasing kinase inhibitor selectivity from medicinal chemistry.

5 Smyth LA, Collins I. Measuring and interpreting the selectivity of protein kinase inhibitors. J. Chem. Biol. 2(3), 131-151 (2009).

6 Huang D, Zhou T, Lafleur K, Nevado C, Caflisch A. Kinase selectivity potential for inhibitors targeting the ATP binding site: a network analysis. Bioinformatics 26(2), 198-204 (2010). discovery. This kinase inhibitor promiscuity can lead to side effects that negatively impact patient quality of life. In the laboratory this promiscuity can greatly hinder our ability to ask questions about the underlying mechanisms of a particular cancer. Because of this, new strategies to increase the selectivity of kinase inhibitors are greatly needed. While there has been significant progress at improving the selectivity of type-I kinase inhibitors, leading to FDA-approved drugs in oncology, kinase inhibitor selectivity is still very much an unsolved problem. While extensive structural optimization can lead to significant gains in selectivity, it is our hope that some of the 'chemical biological' approaches presented above will become modular and predictable parts of the medicinal chemical toolbox to expedite access to new chemical probes that further our fundamental knowledge of cancer biology and lead pharmaceutical compounds with improved toxicological profiles.

\section{Financial \& competing interests disclosure}

The authors' work is supported by SDSU start up and funds from The California State University Program for Education and Research in Biotechnology. The authors have no other relevant affiliations or financial involvement with any organization or entity with a financial interest in or financial conflict with the subject matter or materials discussed in the manuscript apart from those disclosed.

No writing assistance was utilized in the production of this manuscript.

7 Cohen MS, Zhang C, Shokat KM, Taunton J. Structural bioinformatics-based design of selective, irreversible kinase inhibitors. Science 308(5726), 1318-1321 (2005).

8 Liu Y, Bishop A, Witucki L et al. Structural basis for selective inhibition of Src family kinases by PP1. Chem. Biol. 6(9), 671-678 (1999).

9 Liu Q, Sabnis Y, Zhao Z et al. Developing irreversible inhibitors of the protein kinase cysteinome. Chem. Biol. 20(2), 146-159 (2013).

10 Barf T, Kaptein A. Irreversible protein kinase inhibitors: balancing the benefits and risks. J. Med. Chem. 55(14), 6243-6262 (2012).

11 Honigberg LA, Smith AM, Sirisawad M et al. The Bruton tyrosine kinase inhibitor PCI-32765 blocks B-cell activation and is efficacious in models of autoimmune disease and B-cell malignancy. Proc. Natl Acad. Sci. USA 107(29), 13075-13080 (2010).

- Discusses the efficacy of ibrutinib.

12 Jänne PA, Yang JC-H, Kim D-W et al. AZD9291 in EGFR inhibitor-resistant non-small-cell lung cancer. N. Engl. J. Med. 372(18), 1689-1699 (2015).

13 Woyach JA, Furman RR, Liu T-M et al. Resistance mechanisms for the Bruton's tyrosine kinase inhibitor ibrutinib. N. Engl. J. Med. 370(24), 2286-2294 (2014). 
- Seminal findings regarding resistance to ibrutinib through point mutation of targeted cycteine.

14 Gower CM, Chang MEK, Maly DJ. Bivalent inhibitors of protein kinases. Crit. Rev. Biochem. Mol. Biol. 49(2), 102-115 (2014).

15 Brandvold R, Santos SM, Breen ME, Lachacz EJ, Ste ME, Soellner MB. Exquisitely specific bisubstrate inhibitors of c-Src kinase. ACS Chem. Biol. 10, 1387-1391 (2015).

-. Successful example of utilizing bivalent approach to drastically increase the target selectivity of a promiscuous type-I kinase inhibitor.

16 Kwarcinski FE, Steffey ME, Fox CC, Soellner MB. Discovery of bivalent kinase inhibitors via enzyme-templated fragment elaboration. ACS Med. Chem. Lett. 6, 898-901 (2015).

17 Smith DE, Marquez I, Lokensgard ME, Rheingold AL, Hecht DA, Gustafson JL. Exploiting atropisomerism to increase the target selectivity of kinase inhibitors. Angew. Chem. Int. Ed. 54(40), 11754-11759 (2015).

- Seminal precedence for exploiting atropisomer conformation to increase the selectivity of a promiscuous type-I kinase inhibitor.

18 Clayden J, Moran WJ, Edwards PJ, LaPlante SR. The challenge of atropisomerism in drug discovery. Angew. Chem. Int. Ed. Engl. 48(35), 6398-6401 (2009).

- Discusses strategies to deal with atropisomerism in drug discovery.

19 Brown DG, Boström J. Analysis of past and present synthetic methodologies on medicinal chemistry: Where have all the new reactions gone? J. Med. Chem. doi:10.1021/acs. jmedchem.5b01409 (2015) (Epub ahead of print).

20 Maddox SM, Nalbandian CJ, Smith DE, Gustafson JL. A practical Lewis base catalyzed electrophilic chlorination of arenes and heterocycles. Org. Lett. 17, 1042-1045 (2015). 